Steinhauer, Eric W.:

Kritische Anmerkungen zum Zweitveröffentlichungsrecht nach §38 Abs. 1 Satz 3 und 4 UrhG in der Fassung des Bundesratsentwurfes vom 9. Mai 2006

Zuerst erschienen in: Bibliotheksdienst. - Berlin : de Gruyter. - 40 (2006), 6, S. 734-742.

Erstveröffentlichung: $\quad$ 2006-06-01

Digitalisierung: 2013-05-18

ISSN (online): $\quad$ 2194-9646

ISSN (print): $\quad$ 0006-1972

DOI: $\quad 10.1515 / \mathrm{bd} .2006 .40 .6 .734$

[Zuletzt gesehen: 2019-08-14]

„Im Rahmen der hochschulweiten Open-Access-Strategie für die Zweitveröffentlichung identifiziert durch die Universitätsbibliothek IImenau. "

"Within the academic Open Access Strategy identified for deposition by IImenau University Library."

„Dieser Beitrag ist mit Zustimmung des Rechteinhabers aufgrund einer (DFG-geförderten) Allianz- bzw. Nationallizenz frei

zugänglich."

"This publication is with permission of the rights owner freely accessible due to an Alliance licence and a national licence

(funded by the DFG, German Research Foundation) respectively."

DFG

Nationallizenzen 


\section{Kritische Anmerkungen zum Zweitveröffentlichungsrecht nach $\S 38$ Abs. 1 Satz 3 und 4 UrhG in der Fassung des Bundesratsentwurfes vom 9. Mai 2006}

\section{Eric W. Steinhauer}

In der Bundesratsdrucksache (BR-Drs.) 257/1/06 vom 9. Mai 2006 geben der Rechtsausschuss, der Ausschuss für Kulturfragen und der Wirtschaftsausschuss des Bundesrates eine Empfehlung zum Regierungsentwurf für das Zweite Gesetz zur Regelung des Urheberrechts in der Informationsgesellschaft. ${ }^{1}$ In dieser Empfehlung werden viele im Regierungsentwurf vorgesehene Regelungen unter dem Gesichtspunkt der Bildungs- und Wissenschaftsfreundlichkeit kritisiert. ${ }^{2}$ Dabei machen sich die Ausschüsse des Bundesrates die durch viele Wissenschaftsorganisationen, Hochschulen und wissenschaftliche Bibliotheken erhobene Forderung eines möglichst ungehinderten Zugangs zu wissenschaftlicher Information im Sinne von Open Access zu Eigen. ${ }^{3}$

Ein Baustein zur Förderung von Open Access ist die Schaffung eines neuen $\S 38$ Abs. 1 Satz 3 und 4 UrhG/BR-AE ${ }^{4}$. In dieser Norm wird dem Autor eines wissenschaftlichen Textes das vertraglich nicht abdingbare Recht eingeräumt, sein Werk in Form einer Zweitpublikation nach Erscheinen in einer wissenschaftlichen Fachzeitschrift anderweitig zu publizieren.

\section{Geltende Rechtslage der Zweitpublikation}

Derzeit sind Autoren für eine Zweitpublikation ihres Werkes auf § 38 Abs. 1 Satz 2 UrhG angewiesen, sofern vertraglich nichts anderes vereinbart worden ist. ${ }^{5} \mathrm{Nach}$ $\S 38$ Abs. 1 Satz 1 UrhG räumt der Autor eines Zeitschriftenaufsatzes dem Verleger im Zweifel ein ausschließliches Nutzungsrecht ein. Dieses Nutzungsrecht wandelt sich gem. § 38 Abs. 1 Satz 2 UrhG nach Ablauf eines Jahres nach Erscheinen in ein einfaches Nutzungsrecht des Verlegers um. ${ }^{6}$ Der Autor kann dann selbst eine

1 Der Text des Regierungsentwurfs findet sich in BR-Drs. 257/06.

2 Vgl. BR-Drs. 257/1/06, S. I.

3 Vgl. BR-Drs. 257/1/06, S. 8. Dort wird die „Berliner Erklärung“ vom 22. Oktober 2003 ausdrücklich erwähnt.

4 UrhG/BR-AE = Empfehlung der Bundesratsausschüsse zum Urheberrechtsgesetz.

5 Vgl. allgemein dazu Jan Bernd Nordemann, in: Loewenheim, Handbuch des Urheberrechts, München 2003, § 64, Rn. 44; Wandtke/Grunert, in: Wandtike/Bullinger, Praxiskommentar zum Urhberrecht, 2. Aufl., München 2006, § 38, Rn. 6-9.

6 Vgl. Schulze, in: Dreier/Schulze, UrhG, 2. Aufl., München 2006, § 38, Rn. 16. 
Zweitveröffentlichung vornehmen oder Dritten, etwa wissenschaftlichen Bibliotheken, ein weiteres Nutzungsrecht an seinem Werk einräumen. Das gilt nach $\S 38$ Abs. 2 UrhG auch für Beiträge in Sammelwerken, sofern hierfür kein Anspruch auf Vergütung besteht. Damit können in der Regel auch Festschriften- und Handbuchbeiträge nach Jahresfrist anderweitig veröffentlicht werden. ${ }^{7}$ Die in $\S 38$ Abs. 1 Satz 2, Abs. 2 UrhG vorgesehene Möglichkeit der Zweitveröffentlichung für wissenschaftliche Aufsätze ist in der Praxis allerdings nur in den Geistes- und Sozialwissenschaften von Bedeutung. Hier werden regelmäßig keine ausdrücklichen Vereinbarungen bei der Publikation von Aufsätzen geschlossen, so dass die gesetzliche Regelung von § 38 UrhG ohne weiteres zur Anwendung kommt. ${ }^{8}$ Sie spielt bei der retrospektiven Digitalisierung von Aufsätzen durch wissenschaftliche Bibliotheken daher eine wichtige Rolle. Im STM-Bereich jedoch wird das Recht auf Zweitpublikation regelmäßig vertraglich ausgeschlossen. ${ }^{9}$ Nach $\S 38$ Abs. 1 Satz 1 a.E. UrhG gilt die dort getroffene Regelung nämlich nur, "wenn nichts anderes vereinbart worden ist".

\section{Die vorgeschlagene Neuregelung der Bundesratsausschüsse}

Hier setzt der von den Bundesratsausschüssen vorgeschlagene $\S 38$ Abs. 1 Satz 3 und 4 UrhG/BR-AE an. Er gibt dem Autor eines wissenschaftlichen Werkes unter bestimmten Bedingungen ein Recht auf Zweitpublikation. Anderslautende vertragliche Vereinbarungen sind unwirksam, § 38 Abs. 1 Satz 4 UrhG/BR-AE. Im einzelnen lautet die vorgeschlagene Norm:

"An wissenschaftlichen Beiträgen, die im Rahmen einer überwiegend mit öffentlichen Mitteln finanzierten Lehr- und Forschungstätigkeit entstanden sind und in Periodika erscheinen, hat der Urheber auch bei Einräumung eines ausschließlichen Nutzungsrechts das Recht, den Inhalt längstens nach Ablauf von sechs Monaten seit Erstveröffentlichung anderweitig öffentlich zugänglich zu machen, soweit dies zur Verfolgung nicht kommerzieller Zwecke gerechtfertigt ist und nicht in der Formatierung der Erstveröffentlichung erfolgt. Dieses Recht kann nicht abbedungen werden."

7 Vgl. Schmid/Wirth, Urheberrechtsgesetz, Baden-Baden 2004, § 38, Rn. 6; Schulze, in: Dreier/Schulze, UrhG, § 38, Rn. 18.

8 Vgl. Kotthoff, in: Heidelberger Kommentar zum Urheberrecht, Heidelberg 2004, $\S 38$, Rn. 3; Wegner/Wallenfels/Kaboth, Recht im Verlag, München 2004, S. 37 f.

9 Das ist auch in Formularverträgen zulässig, vgl. Jan Bernd Nordemann, in: Loewenheim, Handbuch des Urheberrechts, § 64, Rn. 44. Zur Auslegung entsprechender Vereinbarungen vgl. Schricker, in: Schricker, Urheberrecht, 2. Aufl., München 1999, $\S 38$, Rn. 16. Nach Spautz, in: Möhring/Nicolini, Urheberrechtsgesetz, 2. Aufl., München 2000, § 38, Rn. 1 ist die Vorschrift eine Schutzvorschrift für den Urheber und im Zweifel zu seinen Gunsten anzuwenden. 
Ein gesetzlich verankertes Recht auf Zweitveröffentlichung ist sehr zu begrüßen. Allerdings geben einzelne Voraussetzungen für das neue Recht Anlass zu kritischer Rückfrage. Vorweg ist noch zu erwähnen, dass die von den Bundesratsausschüssen vorgeschlagene Neuregelung in einem Aufsatz von Gerd Hansen im Jahr 2005 vorgestellt und diskutiert worden ist. ${ }^{10} \S 38$ Abs. 1 Satz 3 UrhG/BR-AE stimmt mit der von Hansen angeregten Regelung bis zu den Worten "nicht kommerzieller Zwecke gerechtfertigt ist" wörtlich überein. Die Formatierungserfordernisse für die Zweitveröffentlichung wurden dagegen wohl erst im Rahmen der Ausschussberatungen eingefügt.

\subsection{Berechtigter Autorenkreis}

Nutznießer der vorgeschlagenen Regelung sind nur Autoren von wissenschaftlichen Beiträgen, die im Rahmen einer überwiegend mit öffentlichen Mitteln finanzierten Lehr- und Forschungstätigkeit entstanden sind. Dahinter steht der auch von den Bibliotheken immer wieder betonte Umstand, dass es nicht angehen könne, wenn aus Steuergeldern finanzierte Forschungsergebnisse von der öffentlichen Hand über den Umweg einer teuren Verlagspublikation zurückgekauft werden müssen. ${ }^{11}$

Diese Argumentation rechtfertigt es, den Verlagen, die in gewisser Hinsicht bloße Nutznießer dieser öffentlich finanzierten Ergebnisse sind, ein dauerhaftes ausschließliches Nutzungsrecht zu verwehren. So klar das zunächst scheint, so unklar ist der Anwendungsbereich der gesetzgeberischen Empfehlung. Die öffentliche Lehr- und Forschungstätigkeit bezieht sich wohl auf die dienstliche bzw. arbeitsrechtliche Stellung des Autors. Hier können sich bei subtiler Betrachtung zunächst einige Probleme bei verbeamteten Wissenschaftlern ergeben. Diese werden ja nicht für ihre Tätigkeit bezahlt, sondern lediglich für eine amtsangemessene

10 Vgl. Hansen, Zugang zu wissenschaftlichen Informationen - alternative urheberrechtliche Ansätze, in: GRUR Int. 2005, S. 386 f. (auch online: http://www.gerd-hansen.net /Hansen_GRUR_Int_2005_378ff.pdf [Abruf am 17. Mai 2006], ders., Urheberrecht für Wissenschaftler - Risiken und Chancen der Urheberrechtsreform für das wissenschaftliche Publizieren, in: In die Zukunft publizieren, Herausforderungen an das Publizieren und die Informationsversorgung in den Wissenschaften, Tagungsband zur 11. Jahrestagung der luK-Initiative der Wissenschaftlichen Fachgesellschaften in Deutschland, hrsg. v. Maximilian Stempfhuber, Bonn 2006, S. 9-19 (auch online: http://www.gerd-hansen.net/Hansen_UrhR_fuer_Wissenschaftler.pdf [Abruf am 17. Mai 2006]).

11 Siehe hierzu auch Fournier, Zur Bedeutung von open access für das Publikationsverhalten DFG-geförderter Wissenschaftler, in: ZfBB 52 (2005), S. 235;

Hilty, Das Urheberrecht und der Wissenschaftler, in: GRUR Int. 2006, S. 182. 
Lebensführung alimentiert. ${ }^{12}$ Daher unterfiele alles, was sie wissenschaftlich schreiben, ohne Rücksicht auf ihre konkrete Lehr- und Forschungsaufgabe dem Anwendungsbereich der vorgeschlagenen Neuregelung. Soweit es sich aber um angestellte Wissenschaftler handelt, für die das beamtenrechtliche Alimentationsprinzip nicht zutrifft, wäre demgegenüber immer genau abzugrenzen, ob eine Publikation nun im Rahmen des Anstellungsverhältnisses erfolgt ist oder nicht. Man könnte nun erwägen, den von der Neuregelung zu erfassenden Personenkreis mit dem Merkmal der Zugehörigkeit zum öffentlichen Dienst zu beschreiben. Aber auch dann verbleiben Probleme. Was ist etwa mit Wissenschaftlern, die als externe Doktoranden nicht von der öffentlichen Hand besoldet werden, gleichwohl öffentlich finanzierte Forschungsmittel (Geräte, Bibliotheken) intensiv nutzen, ja ohne diese Mittel gar nicht arbeiten und publizieren können? Und wie sind Wissenschaftler im Ruhestand zu behandeln?

Ausgeschlossen von der vorgeschlagenen Neuregelung sind in jedem Fall an privat finanzierten Instituten tätige Wissenschaftler, nebenberufliche Wissenschaftler und Privatgelehrte. Das ist problematisch, da ihnen die Freiheit des wissenschaftlichen Publizierens, zu der heutzutage auch die Möglichkeit einer Publikation im Internet gehört, im Gegensatz zu ihren aus öffentlichen Mitteln besoldeten Kollegen erschwert wird. ${ }^{13}$ Es scheint vor dem Hintergrund der auch für diese Wissenschaftler unbestritten geltenden Wissenschaftsfreiheit nicht sachgerecht, ihnen eine starke Position gegenüber den mächtigen Wissenschaftsverlagen vorzuenthalten. ${ }^{14}$ Der Gesetzgeber wäre gut beraten, nicht die öffentliche Finanzierung, sondern die Verwirklichung der durch die Wissenschaftsfreiheit grundrechtlich geschützten Publikationsfreiheit zum Anknüpfungspunkt für den neuen § $38 \mathrm{Abs}$. 1 Satz 3 UrhG/BR-AE zu nehmen. ${ }^{15}$ Danach sollten alle wissenschaftlichen Veröffentlichungen unabhängig von der Finanzierung ihrer Autoren und Forschungsmittel von dieser Norm erfasst werden.

12 Vgl. Battis, Bundesbeamtengesetz, 3. Aufl., München 2004, § 83, Rn. 2.

13 Vgl.zur schwierigen Verhandlungslage einzelnen Wissenschaftler gegenüber Verlagen Hilty, Das Urheberrecht und der Wissenschaftler, in: GRUR Int. 2006, S. 186.

14 Das Grundrecht der Wissenschaftsfreiheit gilt für jeden, der wissenschaftlich arbeitet, unabhängig von seiner Zugehörigkeit zu einer Hochschule, vgl. Bethge, in: Sachs, Grundgesetz, 3. Aufl., München 2003, Rn. 209; Jarass, in: Jarass/Pieroth, Grundgesetz, 8. Aufl., München 2006, Art. 5, Rn. 124.

15 Zum Schutz des wissenschaftlichen Publizierens durch Art. 5 Abs. 3 Satz 1 GG vgl. Jarass, in: Jarass/Pieroth, GG, Art. 5, Rn. 122; Kimminich, Das Veröffentlichungsrecht des Wissenschaftlers, in: Wissenschaftsrecht 18 (1985), S. 132; Leuze, Urheberrecht der Beschäftigen im öffentlichen Dienst, 2. Aufl., Berlin 2003, S. 122 f.; Starck, in: von Mangoldt/Klein/Starck, Kommentar zum Gundgesetz, 5. Aufl., München 2005, Art. 5, Rn. 361. 


\subsection{Erfasste Publikationsformen}

Der Ausschussvorschlag bezieht sich nur auf Veröffentlichungen in "Periodika”. Die vorgeschlagene Neuregelung widerspricht damit der bisherigen Wertung des Gesetzgebers in $\S 38$ Abs. 2 UrhG, wonach Beiträge in Sammelwerken den Zeitschriftenaufsätzen gleichgestellt sind, sofern sie nicht vergütet werden. Ein Blick in die Begründung der Bundesratsausschüsse erklärt, warum vor allem an Periodika gedacht worden ist. Dort werden Eckdaten der in Fachkreisen sattsam bekannten Zeitschriftenkrise genannt und die alarmierenden Preissteigerungen zum Anlass für ein gesetzgeberisches Eingreifen genommen. ${ }^{16}$ Die Probleme der Zeitschriftenkrise beziehen sich nicht auf Beiträge in Sammelwerken.

Aus Sicht eines publizierenden Wissenschaftlers aber ist diese Unterscheidung gleichgültig. Wenn man das Recht auf Zweitveröffentlichung nicht so sehr an konkreten Gravamina festmacht, sondern an einer umfassenden und sachgerechten Regelung interessiert ist, sollte man auch bei der Frage der von der Neuregelung erfassten Publikationsformen die Perspektive der publizierenden Wissenschaftler einnehmen und in Sammelbänden erschienene Beiträge entsprechend der derzeit geltenden Rechtslage miteinbeziehen. Allerdings wäre dann zu überlegen, ob für diese Art von Aufsätzen wegen der bei Buchproduktionen anderen Absatzkalkulation nicht eine hemmende Frist von einem Jahr für die Ausübung des Zweitveröffentlichungsrechts sachgerecht wäre.

\subsection{Die Form der Zweitveröffentlichung}

Der wohl problematischste Punkt der vorgeschlagenen Neuregelung ist das Erfordernis, dass die Zweitveröffentlichung nicht in der Formatierung der Erstveröffentlichung erfolgen darf. Nach geltendem Recht kann eine Zweitveröffentlichung, wenn sie rechtlich zulässig ist, durch einfaches Einscannen der publizierten Aufsätze geschehen. Die Neuregelung in $\S 38$ Abs. 1 Satz 3 UrhG/BR-AE verschärft diese Rechtslage für diejenigen Publikationen, an denen Verlagen ein ausschließliches und dauerhaftes Nutzungsrecht eingeräumt worden ist. ${ }^{17}$

Versucht man, die Leistung der Verlage und die Leistung der Autoren an einer Publikation zu trennen, so scheint die vorgeschlagene Regelung auf den ersten Blick sachgerecht zu sein. Das Layout, die Leistung des Verlages, soll nur durch inn ausschließlich verwertet werden, der Inhalt, die Leistung des Autors, darf im Wege einer Zweitpublikation verbreitet werden.

16 Vgl. BR-Drs. 257/1/06, S. 8.

17 Die Formulierung des Vorschlages ist angelehnt an die Modalitäten bei Elsevier, vgl. dazu Dreier, Creative Commons, Science Commons : ein Paradigmenwechsel im Urheberrecht?, in: Perspektiven des Geistigen Eigentums und Wettbewerbsrechts: Festschrift für Gerhard Schricker zum 70. Geburtstag, hrsg. von Ansgar Ohly u.a., München 2005, S. 292, Fn. 34. 
Bei näherem Hinsehen aber ergeben sich einige Fragen. Was ist unter der "Formatierung der Erstveröffentlichung" genau zu verstehen? Sicher soll das einfache Einscannen ausgeschlossen werden. Aber ist es auch verboten, in der Zweitveröffentlichung die Seitenumbrüche der Erstveröffentlichung zu vermerken, um eine Zitierbarkeit der Zweitveröffentlichung zu gewährleisten? Folgt man der Begründung zum vorgeschlagenen $\S 38$ Abs. 1 Satz 3 UrhG/BR-AE, dann wird auch eine nachträgliche Paginierung nicht zulässig sein, denn die anderweitige Veröffentlichung soll, um das Erstverwertungsrecht des Verlegers nicht ungebührlich zu beeinträchtigen, in "nicht zitierfähiger Form" erfolgen. ${ }^{18}$

Diese Regelung ist bibliographischer Unsinn! Zweitveröffentlichungen sind für Wissenschaftler, die mit Literaturzitaten arbeiten, ohne Zitierfähigkeit wertlos. ${ }^{19}$ Der Wissenschaftler könnte sich anhand der Zweitveröffentlichung über den Inhalt eines Beitrages lediglich informieren, um ihn dann, wenn er ihn für eigene Arbeiten zitieren möchte, im Original in der Bibliothek einzusehen oder per Fernleihe zu bestellen.

Hier zeigt sich wiederum, dass der Entwurf nicht konsequent vom Wissenschaftler her denkt. Es scheint so, als sei man allein darauf bedacht, möglichst schonend in die wirtschaftliche Position der Verleger einzugreifen, nicht aber, die Stellung der Wissenschaftler im Publikationsprozess, zu dem nicht nur die Publikation, sondern auch die Rezeption von Publikationen gehört, gegenüber den Verlagen zu stärken. Von daher sollte man wenigstens Anmerkungen über die Originalpaginierung erlauben. Interessant ist übrigens, dass die vorgeschlagene Regelung zur Zweitveröffentlichung in gewisser Weise mittelalterliche Zustände produziert, da nun mindestens zwei, mitunter nicht identische Textvarianten eines Aufsatzes im Umlauf sind, und man bei der Verifizierung von Zitaten fast schon textkritische Methoden anwenden muss, die ansonsten nur bei Frühdrucken oder Handschriften zum Einsatz kommen.

Andererseits können auch die gerechtfertigten Verlegerinteressen nicht ignoriert werden. Der vorgeschlagene Verzicht auf das Originallayout mag bei der kurzen Frist von sechs Monaten für eine zulässige Zweitveröffentlichung angehen. Man sollte ihn aber ergänzen um die Möglichkeit, nach Ablauf von einem bis maximal drei Jahren auch eine Zweitveröffentlichung im Originallayout zu erlauben. Das Interesse der Verleger, das Originallayout eines derart alten Beitrages zu verwerten, dürfte gegenüber dem Interesse des publizierenden Wissenschaftlers, im digitalen Umfeld des Internet seine wissenschaftlichen Beiträge zitierfähig zu dokumentieren, dann in den Hintergrund getreten sein.

18 Vgl. BR-Drs. 257/1/06, S. 9.

19 Zu den Anforderungen an korrektes wissenschaftliches Zitieren vgl. Jele, Wissenschaftliches Arbeiten: Zitieren, München [u.a.] 2003, S. 15 f. 


\section{Folgen für die bibliothekarische Praxis}

Sollte die von den Bundesratsausschüssen vorgeschlagene Regelung Gesetz werden, so hätte dies eine bedeutende Auswirkung auf die bibliothekarische Praxis. Die Hochschulschriftenserver könnten nun eine Fülle von wissenschaftlichen Publikationen bereithalten, auch und gerade im bisher wenig vertretenen STM-Bereich. Es könnte nun tatsächlich gewährleistet werden, dass jede unselbstständige Publikation von Hochschulangehören relativ zeitnah für Forschung und Lehre zur Verfügung steht und zwar unabhängig davon, ob die Hochschule die entsprechenden Zeitschriften abonniert hat. Das ist ein großer Fortschritt.

Problematisch und eine bibliothekarische Herausforderung ist allerdings das Layoutverbot bei Zweitveröffentlichungen. Wenn man nicht eine völlig indiskutable Heterogenität von Dokumenten unterschiedlichster Formatierungen auf dem Server haben will, müssten die Bibliotheken sachgerechte Publikationsformate für eine Zweitpublikation bereitstellen. Neben Formatierungsvorgaben sollte vor allem auf die Zitierfähigkeit geachtet werden, wenn die Zweitveröffentlichung über die bloße Inhaltsinformation hinaus auch für den wissenschaftlichen Publikationsprozess und damit für die Arbeit der Wissenschaftler sinnvoll sein soll.

Unabhängig davon, ob eine Paginierungsanmerkung nach der vorgeschlagenen gesetzlichen Regelung zulässig ist, ist sie praktisch nicht durchführbar. Es wäre ein kaum zu vertretender Aufwand, jedes einzelne Dokument nachzupaginieren. Man sollte sich daher von dem Original vollends lösen und die Zweitveröffentlichung selbst bibliographisch aufwerten. Diese könnte durch die Schaffung einheitlich gestalteter digitaler Postprint-Reihen (mit eigener ISSN) erfolgen. ${ }^{20}$ Die so publizierten Aufsätze wären als solche jedenfalls für die weitere wissenschaftliche Verwertung korrekt zitierbar. ${ }^{21}$ Natürlich sollte man aus bibliographischen Gründen Hinweise zum Ort der Erstveröffentlichung geben. Bemerkenswert ist, dass es hierzu keine gesetzlichen Vorgaben gibt.

\section{Desiderate}

Der vorgelegte Entwurf der Bundesratsausschüsse ist mit Blick auf eine Stärkung von Open Access zu begrüßen. Er ist ein gewaltiger und wichtiger Schritt in die richtige Richtung. Die Begründung zum Entwurf nennt die Ergänzung von

20 Man könnte diese Reihen nach Fachbereichen und Instituten gliedern und hätte so einen transparenten Zugriff auf die Publikationen einer Hochschule.

21 Die Gewährleistung von Zitierbarkeit gehört zu den Aufgaben nachhaltiger Maßnahmen für open access an Hochschulen, vgl. die „Open-Access-Erklärung der HumboldtUniversität zu Berlin“, URL: http://www.edoc.hu-berlin.de/e_info/oa-erklaerung.php [Abruf am 17. Mai 2006]. 
$\S 38$ UrhG einen „Paradigmenwechsel im Bereich wissenschaftlicher Veröffentlichungen". ${ }^{22}$ Das ist nicht übertrieben.

Allerdings ergeben sich bei näherer Lektüre grundsätzliche Bedenken. Der Entwurf argumentiert zu sehr von konkreten Problemen wie Zeitschriftenkrise und sinnvolle Verwendung von Steuergeldern her. Oberste Leitfrage scheint dabei ein möglichst schonender Eingriff in die wirtschaftliche Position der Verlage zu sein. Natürlich ist es richtig, verhältnismäßige Regelungen zu schaffen. Gleichwohl sind, und das ist eine grundsätzliche Kritik, die auszugleichenden Rechtspositionen nicht richtig gewählt. Es geht nicht um die Opposition Steuergelder gegen Verlagsgewinne. Es geht im Urheberrecht letztlich um den Schutz der schöpferischen Persönlichkeit, also um die Autoren. ${ }^{23}$ Im Bereich der wissenschaftlichen Publikationen ist überdies das in Art. 5 Abs. 3 Satz 1 Grundgesetz (GG) gewährleistete Grundrecht der Wissenschaftsfreiheit zu beachten. Der Gesetzgeber sollte den von ihm zu leistenden Interessenausgleich daher nicht von den Steuermitteln, sondern von der Wissenschaftsfreiheit der Autoren her denken. Die vorgeschlagene Regelung in $\S 38$ Abs. 1 Satz 3 UrhG/BR-AE müsste somit die grundrechtliche Position der Wissenschaftler und das wirtschaftliche Interesse der Verlage zum Ausgleich bringen. Das Interesse der Verlage ist vor allem durch angemessene Fristen zu wahren, um ihnen eine wirtschaftliche Verwertung der verlegten Publikationen zu ermöglichen. Das Interesse der Autoren aber liegt bei einem durch das Grundrecht der Wissenschaftsfreiheit gewährleisteten wissenschaftsadäquaten Publikationswesen. ${ }^{24}$ Dazu gehört auch die freie und ungehinderte Verbreitung von Veröffentlichungen im digitalen Umfeld des Internet. Aus Sicht der Wissenschaftler müssten die Einschränkungen auf öffentlich finanzierte Forschung und auf Zeitschriftenaufsätze entfallen; die Layoutfrage wäre im Sinne einer wissenschaftsgemäßen Zitierbarkeit zu lösen.

22 BR-Drs. 257/1/06,S. 8.

23 Vgl. nur Rehbinder, Urheberrecht, 13. Aufl., München 2004, § 3, Rn. 26.

24 Vgl. Leuze, Die urheberrechtliche Stellung des Professors, insbesondere dargestellt am Beispiel wissenschaftlicher Sprachwerke, in: Wissenschaftsrecht 34 (2001), S. 160 f.; Pernice, in: Dreier, Grundgesetz, 2. Aufl., Tübingen 2004, Art. 5 III, Rn. 28, der vor allem die Bedeutung wechselseitiger Information und Kommunikation hervorhebt. Daraus ergibt sich, dass ein Publikationssystem, das den Fluss von Information zunehmend hindert, nicht dem Leitbild der grundrechtlich geschützten Wissenschaftsfreiheit entspricht. Wenn der einzelne Wissenschaftler ein offenes Publikationswesen monopolistischen Verlagen gegenüber nicht mehr durchsetzen kann, ist es bedenkenswert, aus dem Grundrecht der Wissenschaftsfreiheit eine Verpflichtung des Gesetzgebers abzuleiten, gravierende Fehlentwicklungen zu korrigieren. 
Auch wenn die vorgeschlagene Regelung in $\S 38$ Abs. 1 Satz 3 und 4 UrhG/BR-AE durchaus verbesserungsfähig ist, ist sie sehr zu begrüßen. Für die Bibliotheken bedeutet sie keine Verschlechterung, da die bisherige Rechtslage in $\S 38$ UrhG unangetastet bleibt. Für den Bereich der ohne ausdrückliche Vereinbarung publizierten Aufsätze kann die Praxis des retrospektiven Digitalisierens durch schlichtes Einscannen fortgeführt werden. Für die übrigen Aufsätze, vor allem aus dem STM-Bereich, wären neue Dienstleistungen nachhaltigen wissenschaftlichen Publizierens im digitalen Kontext zu entwickeln. 